This item was submitted to Loughborough's Research Repository by the author.

Items in Figshare are protected by copyright, with all rights reserved, unless otherwise indicated.

\title{
Eliminating end effects for theoretical panel buckling with FEM
}

PLEASE CITE THE PUBLISHED VERSION

http://www.aiaa.org/content.cfm?pageid=2

\section{PUBLISHER}

(c) American Institute of Aeronautics and Astronautics (AIAA)

VERSION

AM (Accepted Manuscript)

LICENCE

CC BY-NC-ND 4.0

\section{REPOSITORY RECORD}

Fenner, Patrick, and Andrew Watson. 2011. "Eliminating End Effects for Theoretical Panel Buckling with FEM". figshare. https://hdl.handle.net/2134/8692. 
This item was submitted to Loughborough's Institutional Repository (https://dspace.lboro.ac.uk/) by the author and is made available under the following Creative Commons Licence conditions.

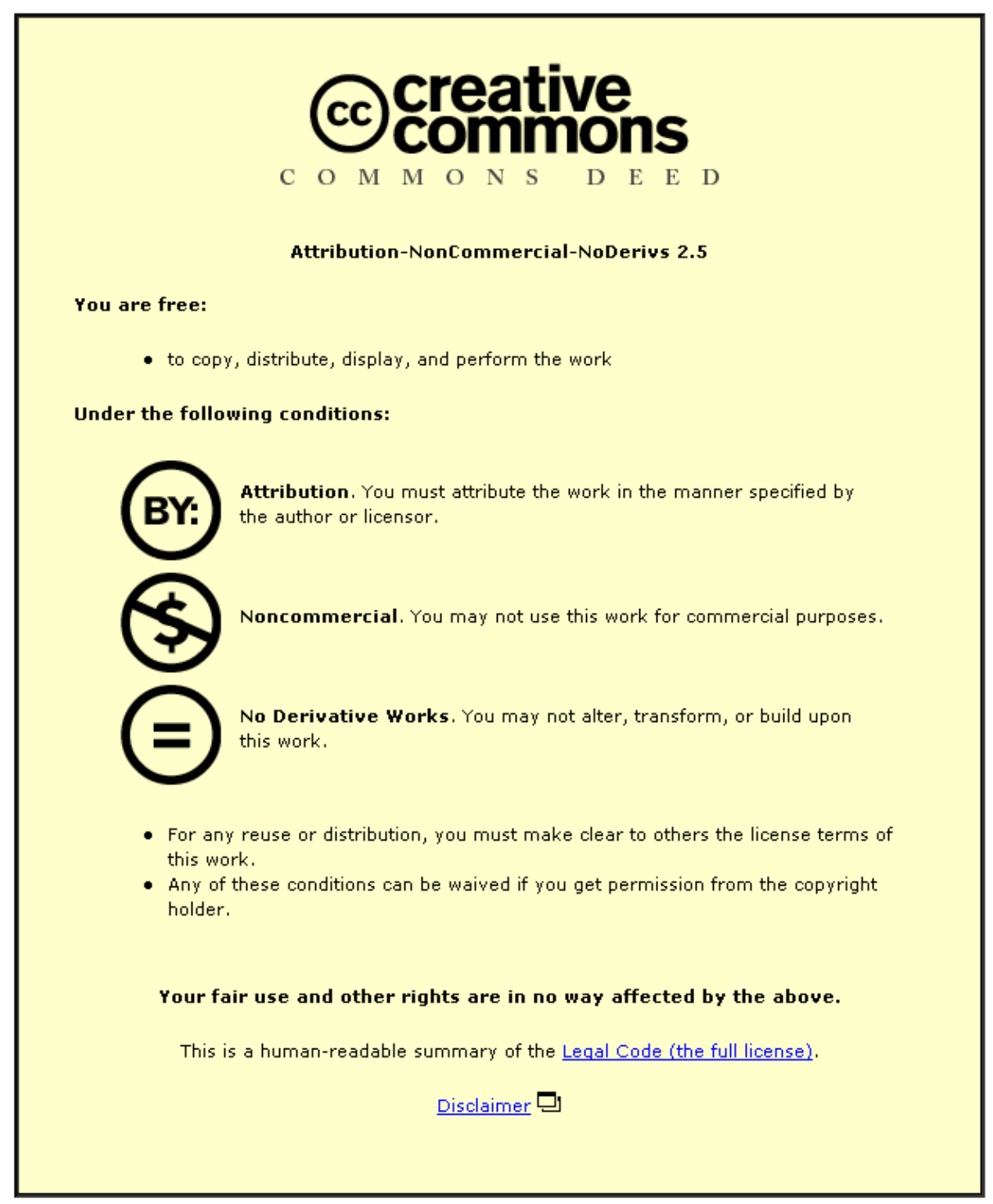

For the full text of this licence, please go to: http://creativecommons.org/licenses/by-nc-nd/2.5/ 


\title{
Eliminating End Effects for Theoretical Panel Buckling with FEM
}

\author{
Patrick Fenner ${ }^{1}$ and Andrew Watson ${ }^{2}$ \\ Loughborough University, Leicestershire, LE11 3TU, UK
}

Document Version: 1.0, released March 2010.

The theoretical buckling performance of thin walled panels under compression can be predicted using classical plate theory (CPT) and using tools such as the VICONOPT, which uses the finite strip method, and Abaqus, a finite element modelling program (FEM). VICONOPT is much more computationally efficient than FEM, and is able to optimise panel design for buckling and pseudo-postbuckling performance. This work forms part of a larger project to use VICONOPT to optimise panel designs that are allowed to buckle in a stable manner below the maximum allowed loading. Because VICONOPT can only make a first approximation of postbuckling performance, a method is required to validate the optimisation results against a more accurate method.

VICONOPT assumes an infinitely long panel, and while FEM can be used to predict both buckling and postbuckling performance of finite sized panels. The authors were unable to find published instances of boundary condition calculations or a method that could be used to verify VICONOPT's theoretical determinations with an infinite length panel that was able to predict all possible buckling modes; without additional invalid buckling modes. And they were unable to find a program that was able to predict mode jumping in the post-buckling range. This paper presents three FEM models for the finite element modelling of the buckling and post-buckling performance of infinite length thin walled stiffened panels; a single bay, a double half-bay and a quad half-bay model. The quad half bay model is shown to be the ideal model as all wavelengths of buckling are permitted and thus enables the calculation of buckling performance of theoretical panels accurately using FEM, and the accurate post-buckling and collapse performance, including mode jumping.

\section{Nomenclature}

$\lambda_{x}=$ Longitudinal half-wavelength.

$\lambda_{y}=$ Transverse half-wavelength.

$\sigma \quad=$ Stress.

$\varepsilon=$ Strain.

$A=$ Cross sectional area.

$B \quad=$ Panel width (equal to $b$ for a plate).

$D \quad=$ Flexural rigidity.

$E \quad=$ Young's modulus.

$N \quad=$ Total applied end load.

$N_{x} \quad=$ Area averaged, axial load per unit width.

$P \quad=$ Panel axial stiffness $(P=\delta F / \delta d)$.

$P^{*} \quad=$ Pre-buckling axial stiffness.

$a \quad=$ Plate length (equal to $l$ if panel is prismatic).

$b \quad=$ Plate width (normally equal to stiffener pitch).

\footnotetext{
${ }^{1}$ Research Student, Aeronautical and Automotive Engineering, P.E.Fenner@lboro.ac.uk

${ }^{2}$ Lecturer, Aeronautical and Automotive Engineering, A.Watson@lboro.ac.uk, AIAA Senior Member 


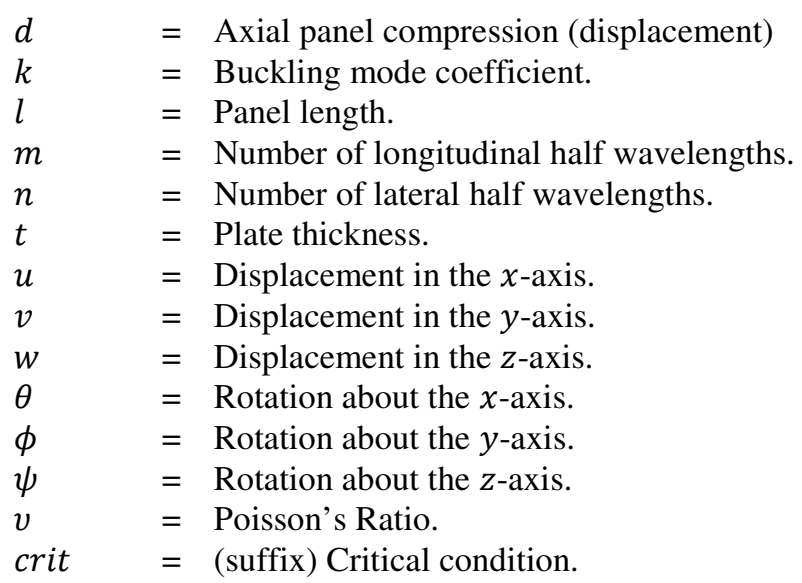

All formulas and calculations use S.I. units exclusively.

\section{Introduction}

$\mathrm{M}^{\circ}$ ODERN aircraft wings are thin walled structures composed of ribs, spars and stiffened skins. For civil aircraft, the top skin is subject under aerodynamic loading to compressive forces that can cause buckling instability. As a simplification for analysis, the top wing surface can be divided into a series of rectangular, flatskinned, stiffened panels with simply supported (SS) boundary conditions.

Classical plate theory suggests that a plate will buckle into a mode, where the shape is dependent upon both aspect ratio and applied load ${ }^{1}$. Equation 1 defines the critical load per unit width for an axially loaded, simply supported plate (width $b$ and length $a$ ), the result is a mode shape made up of a continuous pattern of a number of half sine waves (of length $\lambda$ ) in each orthogonal direction ( $m$ being the quantity running longitudinally and $n$, laterally).

Equation 1 shows that, as with column buckling, where there are alternative buckling mode shapes that could theoretically occur; there is a set of possible mode shapes for a plate buckling such that $m=a / \lambda_{x}$ and $n=b / \lambda_{y}$ are both positive integers. It is the buckling mode that requires least load to occur that is critical; however, unlike for column buckling, the opportunity exists for the mode shape to jump to a different mode at a higher applied load due to postbuckling stability.

where,

$$
N_{x}=\frac{k \pi^{2} D}{b^{2}}
$$

$$
D=\frac{E t^{3}}{12\left(1-v^{2}\right)}
$$

and,

$$
k=\left(\frac{m b}{a}+\frac{n^{2} a}{m b}\right)
$$

Where $D$ represents the Flexural Rigidity of the plate, $k$ the buckling mode coefficient, which is dependent on buckling mode shape, and $t$ is the plate thickness. $E$ is Young's Modulus and $v$ is Poisson's Ratio.

A panel is a collection of plates, consisting of a flat skin plate that has a number of stiffeners, each a structure of thin plates, attached to it. While not all aircraft skin panels are such, this paper (and the associated research) deals exclusively with panels that are prismatic along the loading direction. A selection of typical cross sections is shown in Figure 1. When a panel buckles initially, the bay (single plate between junctions) that has the lowest critical buckling load deforms into its buckled shape, and this deformation is propagated through adjoining bays to form a buckled shape across the whole panel. Because of the complexity of this process, it is normal to use a computer program to predict this occurrence. 


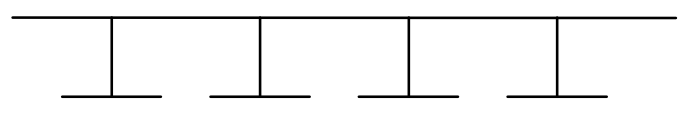

a)

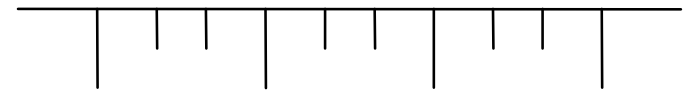

c)

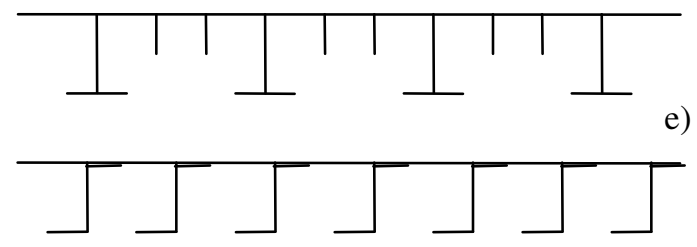

g)

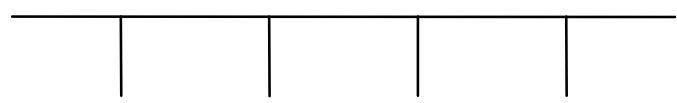

b)

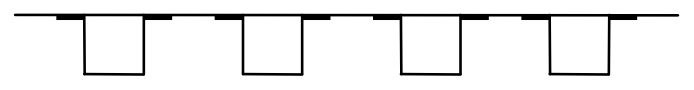

d)

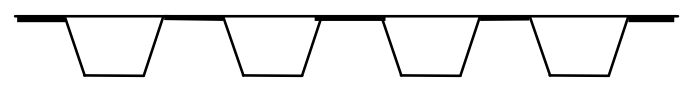

f)

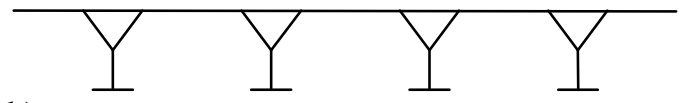

h)

Figure 1: Thin-Walled Panel Cross-Sections

For panel buckling, there are three main types of buckling mode classification characterised by the cross section diagrams in Figure 2.

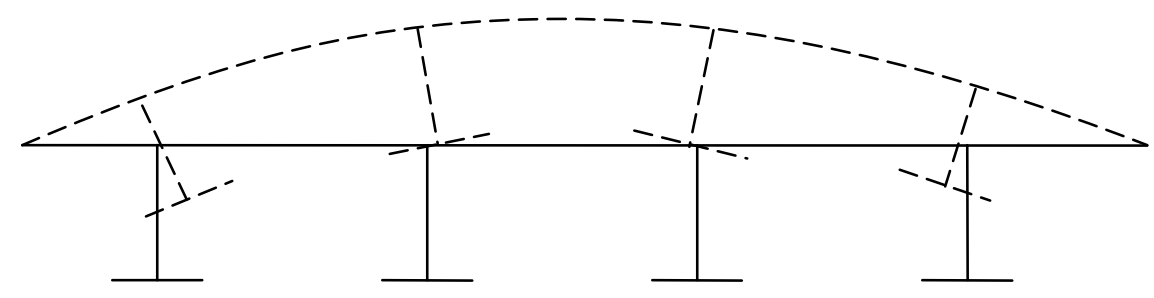

a.) Overall Buckling Mode

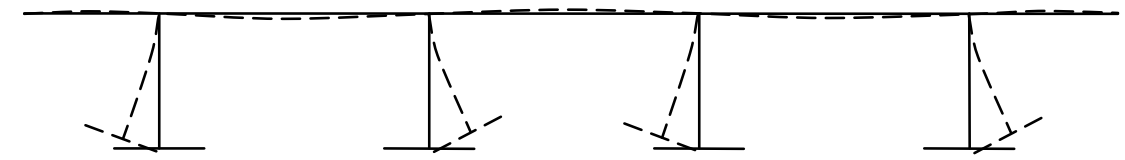

b.) Torsional Buckling Mode

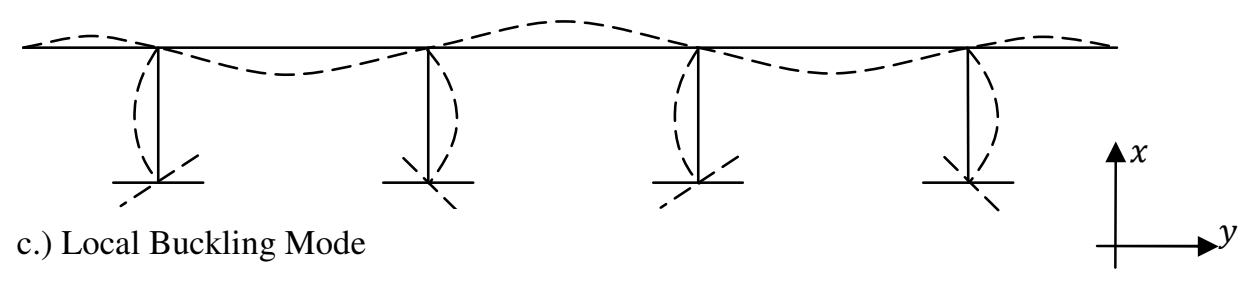

Figure 2: Buckling Modes of a Stiffened Panel

An overall buckling mode is inter-bay, so all the stiffener/skin and stiffener/web line junctions move out-ofplane to the skin. This is similar to plate, where the additional structure of the stiffeners has not qualitatively affected the resulting mode shape. These modes have poor postbuckling performance and lead directly to panel failure and collapse. 
Torsional buckling modes are characterised by small skin displacement with skin/stiffener line junctions remaining linear and stiffener web/flange line junctions deflecting; buckling is intra-bay in the skin, and inter-bay in the stiffeners. These buckling modes can have a positive postbuckled stiffness in some circumstances. ${ }^{2}$

Local buckling modes show no deflection of the skin/stiffener and stiffener web/flange line junctions along the length of the panel to first order accuracy. All the deformation occurs intra-bay, with only rotation at the bay junctions. Because the bay junctions remain linear, load is transferred after buckling to these areas, allowing a significant postbuckling reserve of strength and positive postbuckled stiffness.

Unlike buckling in columns, the initial buckling load of a panel can be different from the collapse load. When the initial buckling mode has a stable post-buckling stiffness, the load can be increased and the panel will "jump" into higher energy modes, where the stiffness for each mode will be less than the one preceding it, until the panel reaches an unstable buckling mode and collapses or the plate experiences a material failure.

\section{Modelling Buckling Modes}

For the analysis of plate structures, two main methods exist, the Finite Strip Method (FSM) ${ }^{3}$ and the more general Finite Element Method (FEM). ${ }^{4}$ The aim of this paper is to reproduce an equivalent FEM model of a FSM model.

FSM is a method of calculating the response of plates subjected to axial loads, based on classical plate theory; FSM assumes continuous mass and stiffness to produce a mathematically exact result for any longitudinally invariant plate structure that can be described by a number of rectangular cross section plates. The application of FSM assumes that the buckling mode of a plate structure can be described by a sinusoidal response of an infinitely long plate, where this is a sum of specific half-wavelengths that factor exactly to the described panel length and as a function of the applied loading.

VICONOPT (VIPASA with CONstraints and OPTimization) is a FORTRAN 77 computer program that incorporates the earlier programs VIPASA (Vibration and Instability of Plate Assemblies including Shear and Anisotropy) and VICON (VIPASA with CONstraints). As an application of FSM it covers any prismatic plate assembly, composed of anisotropic plates each of which can carry any combination of uniformly distributed and longitudinally invariant in-plane stresses. It can be used as either an analysis or an optimum design program. The analysis principally covers the calculation of eigenvalues, i.e., the critical load factors in elastic buckling problems or the natural frequencies in undamped vibration problems, and uses an exact stiffness approach resulting from solution of the governing differential equations of the constituent members. In the VIPASA analysis, ${ }^{5}$ the deformation is assumed to vary sinusoidally to infinity in the longitudinal direction, yielding exact stiffness matrices whose elements are transcendental functions of the load factor or frequency and the axial half-wavelength, $\lambda$, of the deformation. The resulting transcendental eigenproblem requires an iterative solution which is performed using the Wittrick-Williams algorithm. ${ }^{6}$

To validate any optimised panels designed using VICONOPT, the FEM model has to be able to model the initial buckling, postbuckling and collapse of a theoretical, infinite length, simply supported panel, equivalent to the conditions that VICONOPT assumes. Any validation model must be able to recreate any buckling mode that could occur in the theoretical structure, without introducing any invalid modes that only occur as a result of the modelling method.
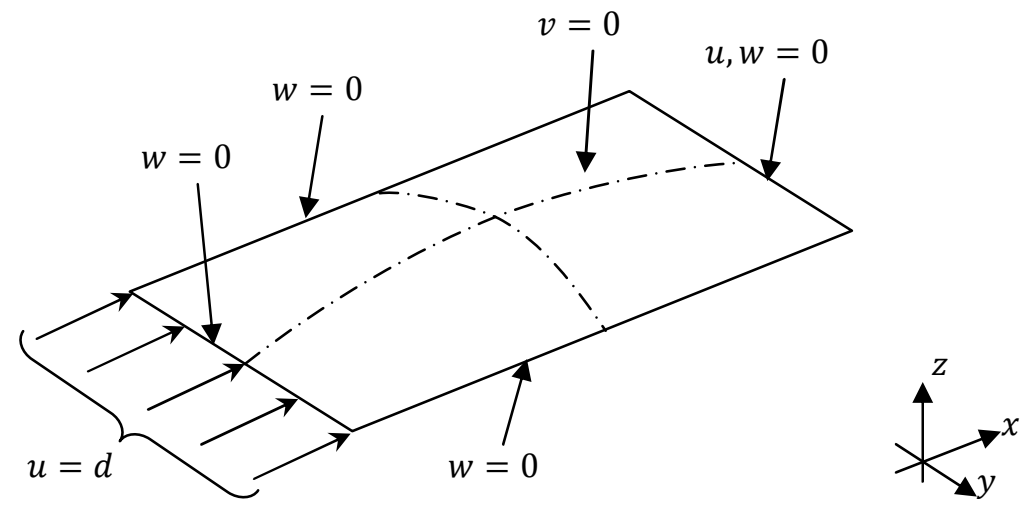

Figure 3: Boundary Conditions for a Flat Plate 
Using Abaqus, it is straight forward to meet these requirements for a plate. In a 2D shell model, simple supports can be applied to each edge and load or displacement applied to the axial ends as shown in Figure 3. Only the loading method needs particular attention. Before initial buckling, load is applied with constant stress across the area, but in the postbuckled region, to keep the panel ends linear (and adhere to the conditions used by classical plate theory) the stress distribution changes inversely to the out-of-plane deflection. At the $y$-position where out-of-plane deflection is greatest along the length, stress in the $x$-axis is reduced (and may become reversed); the load that would be carried by this area is distributed towards the places where there is less out-of-plane deflection, like the lateral edges (for panels in local buckling modes, load is transferred towards the bay junctions). To remove the requirement to adjust the load manually, one end of the plate model has a fixed, zero longitudinal deflection and constant axial deflection is applied to the other end, so the load will be vary automatically in post buckling. The distribution of axial stress $\left(\sigma_{x}\right)$ at the displaced end is shown in Figure 4 for selected $d / d_{\text {crit }}$ levels.

The calculated results are for a $1 \mathrm{~m}$ square plate with $b / t$ ratio of 100 , made of aluminium with a Young's Modulus of $72.4 \mathrm{GPa}$, and a Poisson's Ratio of 0.3. All Abaqus results presented in this paper use S4R elements with reduced integration, default hourglass control, and finite membrane strains.

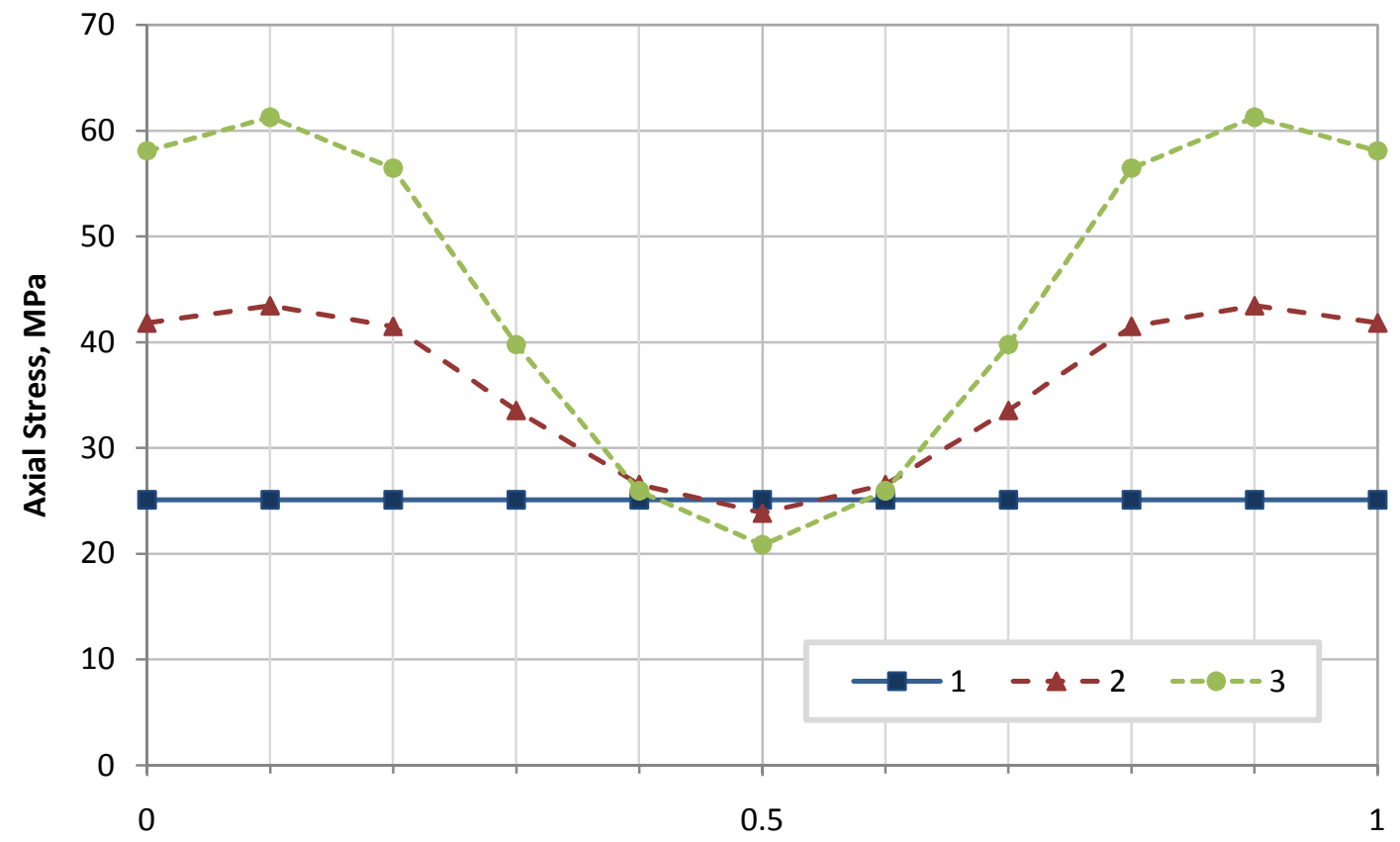

End Node $y$-position, normalised to width

Figure 4: Postbuckling Axial Stress Distribution for Multiples of Critical Displacement.

\section{Modelling Panel Buckling}

For comparison, this paper models the support and loading conditions for a previously investigated panel. Taken from the Dawe and Wang paper ${ }^{7}$ the "NASA Example 4" panel was used for this analysis. The panel is a relatively simple stiffened panel, with six blade stiffeners, the material is isotropic, and the panel is only loaded with axial compression. The panel has been previously analysed by Dawe and Wang, ${ }^{7}$ where postbuckling performance was analysed using non-linear spline FSM; Peskham and Dawe, ${ }^{8}$ where buckling and postbuckling performance was predicted using S-a FSM; and Stroud, Greene and Anderson ${ }^{9}$ who conducted an FEM study of performance, with all studies in close agreement of panel performance. Panel cross sectional geometry is described in Figure 5, where $l=B=0.762 \mathrm{~m}, E=72.4 \mathrm{GPa}$ and $v=0.32$.

For panel modelling, the infinite length model is more difficult to construct, the addition of stiffeners means the panel boundaries are two dimensional and this makes the support conditions non-trivial. Stiffeners meet the longitudinal boundary and mean loading and support conditions used for the plate are no longer valid. Applying displacement to the skin only will not load the structure about the neutral axis and will cause a bending moment that will affect both the buckling and postbuckling performance. 


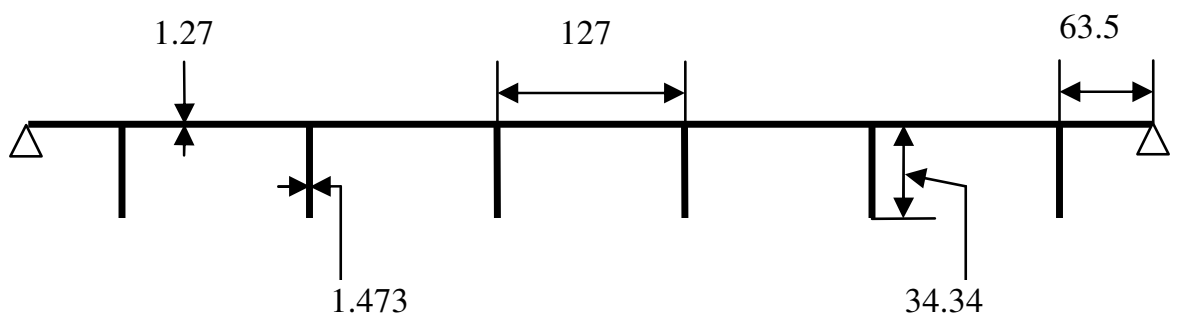

Figure 5: Cross Section of "NASA Example 4" Panel. Centreline Dimensions in mm.

\section{A. Single Bay Model}

The starting point for panel modelling is a single bay model. As in the case of the plate, the skin is simply supported at all sides, at $x=0, l$ and $y=0, B ; w=0$ and constrained laterally along the centreline, where at $y=B / 2 ; v=0$. Axial loading and constraint, though, need particular attention.

As described above, applying displacement or load at a line away from the axial centroid will induce a bending moment to the panel; so for accuracy, one must be applied at, or averaged across, the centroid of area. The panel cannot be loaded at each end with constant stress because, as with the panel model, stress will be redistributed across the component plates based of buckling mode. Because the aim of the analysis is to determine the postbuckling mode and performance, this cannot be known beforehand.

Displacement control could be applied to the end section, but not without some caveats. If displacement is applied only to the axial centroid, this will cause a stress concentration around this line. This will cause a nonuniform stress distribution before buckling that cannot redistribute over the cross section after buckling. The result of this loading case would be a volume at each end of the panel where the stress distribution through the material is not representative of the infinite length case.

To allow the correct distribution of stress, a constant displacement could be applied to the whole cross section, as in the plate example above and shown for the NASA Example 4 panel in Figure 6. This is representative of "diaphragm end conditions" as described by Dawe and Wang, ${ }^{7}$ where in the plate's local displacements at $x=0$; $u=d$; at $x=l ; u=0$; and at $x=0, l ; w=0$ and $v \neq 0$; or "component plates are simply supported for out of plane behaviour and are free to expand in their planes". 7
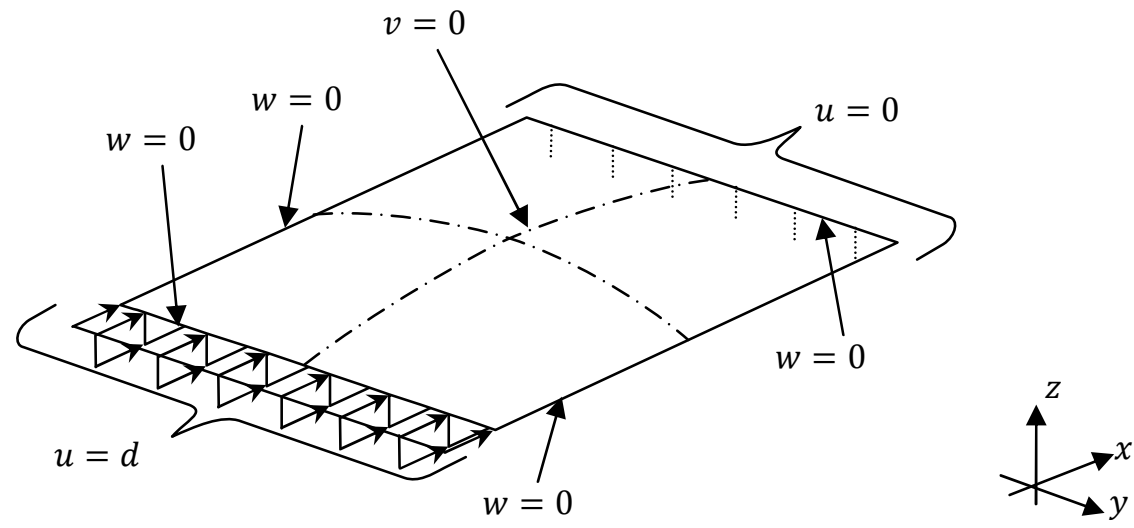

Figure 6: Single Bay Model and Boundary Conditions

While a single bay model is sufficient for most panel buckling studies, it is fundamentally flawed because it cannot allow all possible buckling mode shapes; it will give erroneous results for some buckling studies and is completely unsuitable for postbuckling. For local and torsional buckling modes, at the lateral nodal lines, the panel cross section will scale in-plane, due to Poisson's effect, but will not warp out-of-plane. For these two buckling modes types, the application of constant displacement will correctly identify the buckling behaviour, because the axial ends will always be nodal lines as (from CPT) $m=a / \lambda_{x}=l / \lambda_{x}$ where $m$ is always an integer. This will mean 
that buckling calculations for panels that have local or torsional modes critical, like the NASA Example 4 panel in this paper, will be correct for these mode types.
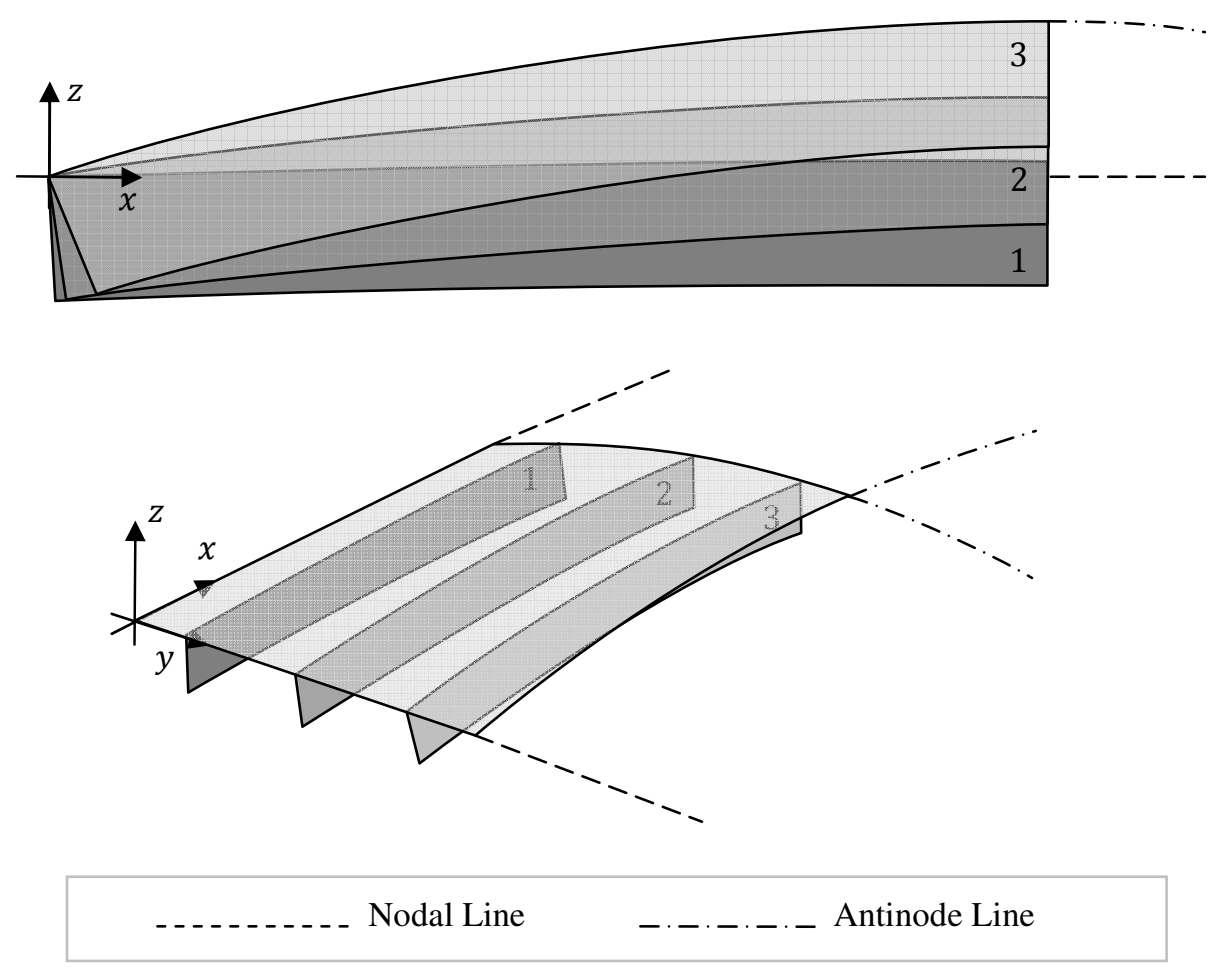

Figure 7: End Rotation of Stiffeners for an Overall Mode

For overall modes in panels, the skin deflects out-of-plane in all locations except at the nodal lines because the skin plates are constrained to $w=0$ at $x=0, l$ and at $y=0, B$. However, at the nodal lines, the stiffeners will rotate about the $y$-axis, and this rotation will be the same as the $y$-axis rotation of the adjoining skin. This means for an $m=1, n=1$ mode, the rotation of the central two stiffeners will be greater than the rotation of the outer stiffeners (see Figure 7) meaning that while the cross section scales for Poisson's effect, it also warps and becomes nonplanar. Because of this, any constant displacement conditions applied to the boundary will not correctly predict the simply supported response for overall modes. Instead, the FEM model will only predict the fixed end responses; and because fixed end responses have higher critical axial forces than simply supported modes, these end conditions will miss collapse modes.

\section{B. Double Half-Bay Model}

Because, if the panel carries no shear, modes in an infinite length panel must be symmetric about some line, it is possible to load the panel with constant displacement on that line. The buckling conditions predicted by VICONOPT and CPT suggest that buckling modes will be a sinusoidal pattern and therefore symmetric about all antinodes (see Figure 7). The addition of stiffeners will not affect this relationship, meaning that it is possible to load a panel with constant displacement at a known antinode position.

In reality, the determination of how to load a panel at an antinode becomes easier to make. Only the critical overall mode need be considered for loading because overall modes do not have positive postbuckling stiffness, so an overall mode in postbuckling will lead to a panel collapse without entering a higher-load mode. So for the NASA Example 4 panel, which has a $\lambda_{x}=l / 1$ mode as the lowest overall mode; the panel could be loaded at the mid-span (as in Figure 8). Because both the nodes and antinodes for the local modes are both planar, is it possible for all integer $m$-modes to form correctly, so the model accurately predict all infinite length buckling modes and loads up to, and including the $m=1$ overall load in order of criticality. 

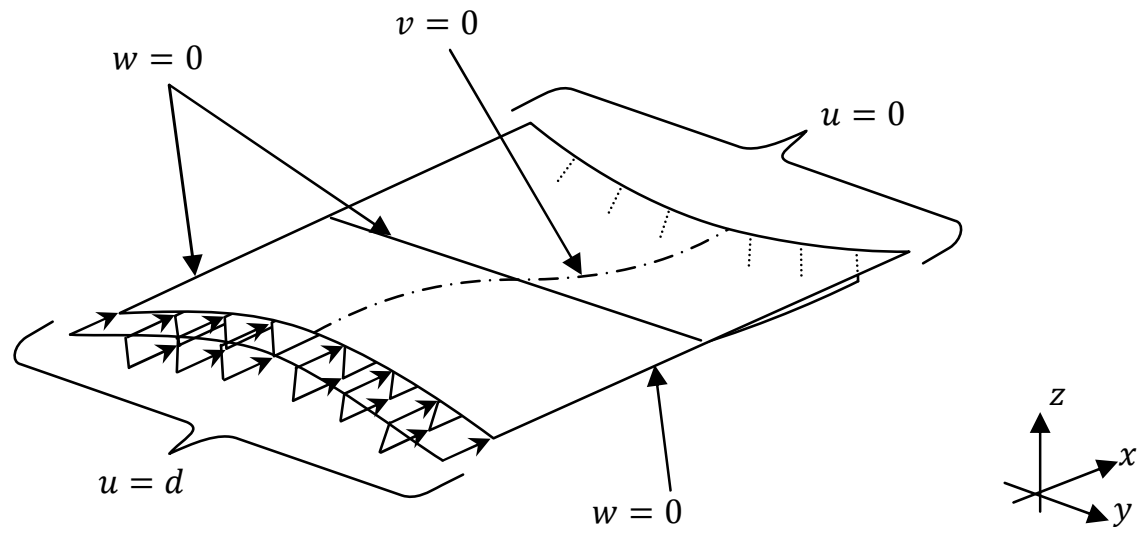

Figure 8: Double Half-Bay Model and Boundary Conditions

\section{Elimination of End Effects}

As it stands, the FEM double-half bay model will correctly identify all the infinite buckling modes of the NASA Example 4 panel, but it will also give rise to a number of additional mode shapes, similar to additional modes that exist the single bay model without additional constraints.
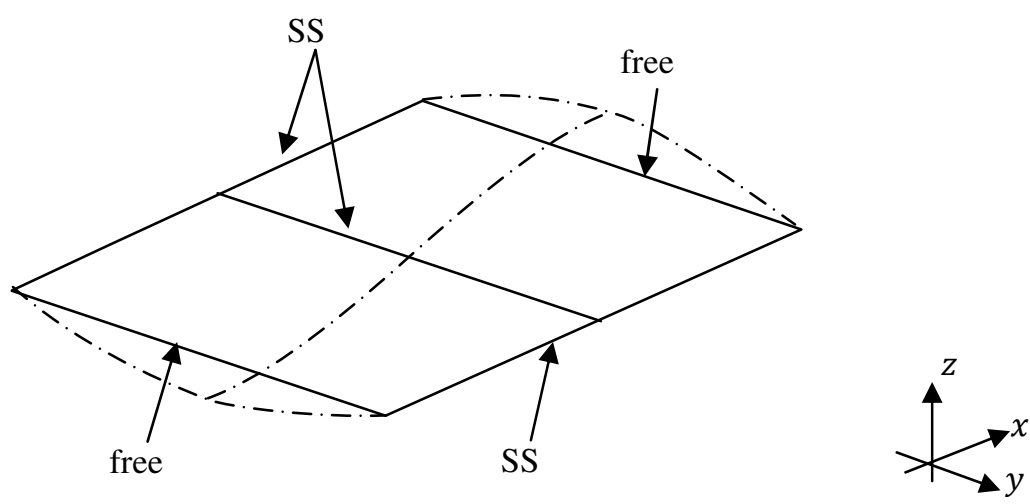

Figure 9: Double free-SS-SS-SS Panel

The double half-bay model is essentially a pair of free-SS-SS-SS panels, loaded in the free-SS direction as shown in Figure 9. Plates that are loaded along an unsupported edge will have buckling modes that have large out of plane deflections at that edge, which will not occur in infinite length plates. This is confirmed by FEM testing and an example is shown in Figure 10b. Similarly for the single bay model, unless the stiffeners are restricted in their local out-of-plane direction, as prescribed by the Dawe \& Wang's specification of diaphragm ends, ${ }^{7}$ buckling modes are possible where the stiffener ends deform locally with minimal global buckling of the panel. An example is shown in Figure 10a.

To stop these modes, which do not occur in infinite length panels, from being a result in the model, some link could be made between the axial free ends. This would link the stresses and strains from one end of the model to another, effectively making the model represent an infinite length "loop". 


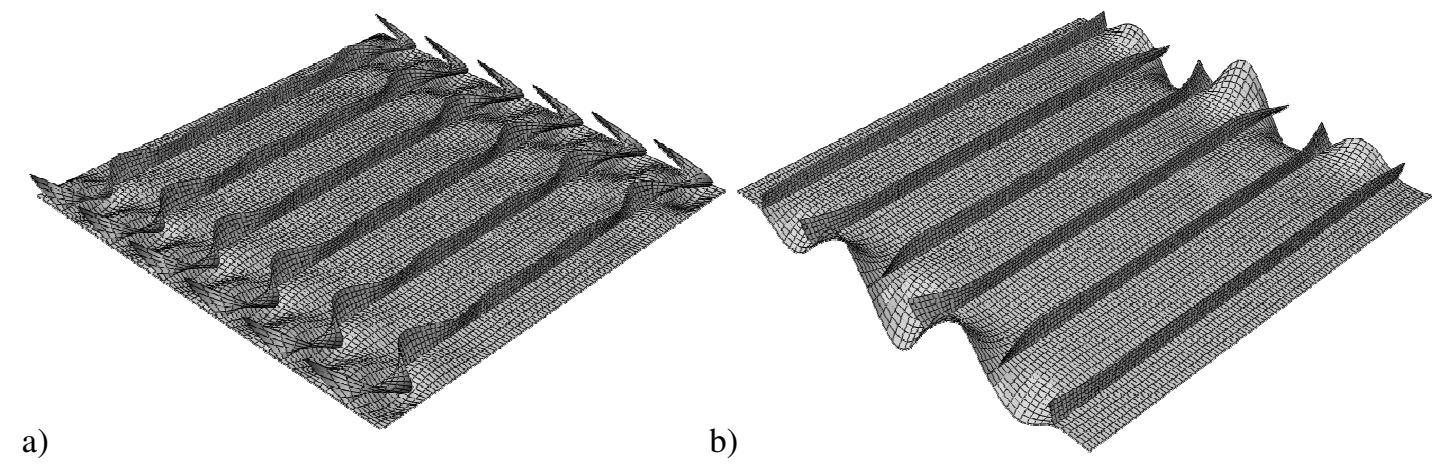

Figure 10: Erroneous Buckling Modes Shapes Showing End Effects, a) Single Bay and b) Double Half-Bay

The axially loaded and constrained ends of the model are known to be planar at all times, so simply linking displacements and rotations at equivalent end nodes will be sufficient to create and infinite length condition, except that there are two possible end cases. For modes where $m$ is even, the end will be at a nodal position and therefore the junction to the "next" panel length would be anti-symmetric and identical to the opposite end. However, for modes where $m$ is odd, the panel end will be at an anti-node, so the join will be symmetric to the next panel bay but vertically opposed to the opposite end; see Figure 11. These relationships can be described by the following equations for each end node pair.

So, for equivalent nodes,

For odd modes $(m=1,3,5, \ldots)$ :

$$
v_{o}=-v_{l} \quad w_{o}=-w_{l} \quad \theta_{0}=-\theta_{l} \quad \phi_{o}=-\phi_{l}=0 \quad \psi_{o}=-\psi_{l}=0
$$

And for even modes $(m=2,4,6, \ldots)$ :

$$
v_{o}=v_{l} \quad w_{o}=w_{l} \quad \theta_{0}=\theta_{l} \quad \phi_{o}=\phi_{l} \quad \psi_{o}=\psi_{l}
$$

And $u_{0}=d, u_{l}=0$ for both odd and even modes.

Because it is not possible to assign both conditions to each end pair, the effect of adding the constraints to remove the end effects is that now either only odd or only even modes can be calculated in a single solution. This is similar to the single bay model, where even though all modes end at a nodal line, even-modes will be anti-symmetric and continuous end-to-end, while odd-modes will be anti-symmetric and vertically reversed in the adjoining bay. The restriction of buckling modes for single bay models is more subtle than for the double half-bay model, where the buckling and postbuckling properties are affected and give rise to buckling modes that appear appropriate but do not represent the real properties of the infinite length panel. An example is described in the results below. 


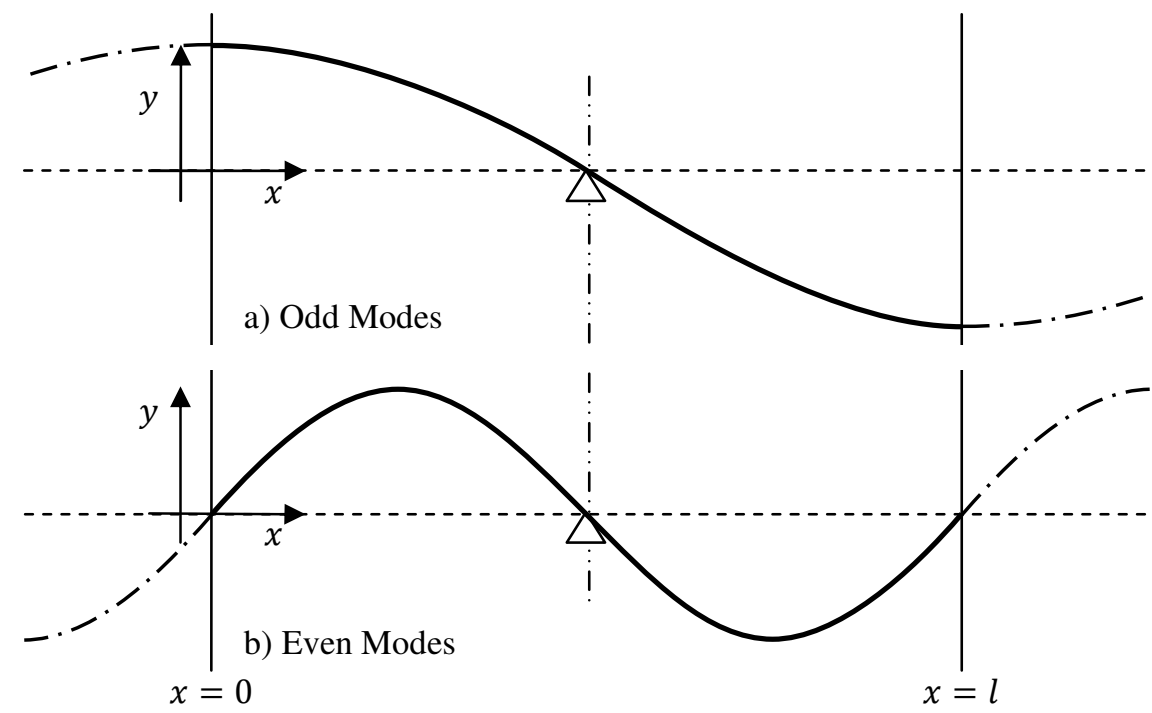

Figure 11: Comparison of Odd and Even Longitudinal Modes.

\section{Quad Half-Bay Model}

To resolve the mode separation problem, the length of the model can be doubled, as shown in Figure 12. This means that all modes for a single length panel become even modes of the double length panel; so for all valid modes, the end connections are anti-symmetric and continuous for the end-to-end connections.

So, for equivalent nodes,

For odd modes $\left(l / \lambda_{x}=1,3,5, \ldots\right)$ :

$$
v_{o}=v_{2 l} \quad w_{o}=w_{2 l} \quad \theta_{0}=\theta_{2 l} \quad \phi_{o}=\phi_{2 l} \quad \psi_{o}=\psi_{2 l}
$$

And for even modes $\left(l / \lambda_{x}=2,4,6, \ldots\right)$ :

$$
v_{o}=v_{2 l} \quad w_{o}=w_{2 l} \quad \theta_{0}=\theta_{2 l} \quad \phi_{o}=\phi_{2 l} \quad \psi_{o}=\psi_{2 l}
$$

This model suffers from increased solution time as the number of elements is doubled, but accuracy is greatly increased over the double half-bay model by allowing correct solution of, and mode jumping between, all valid modes for the theoretical, infinite length panel. 


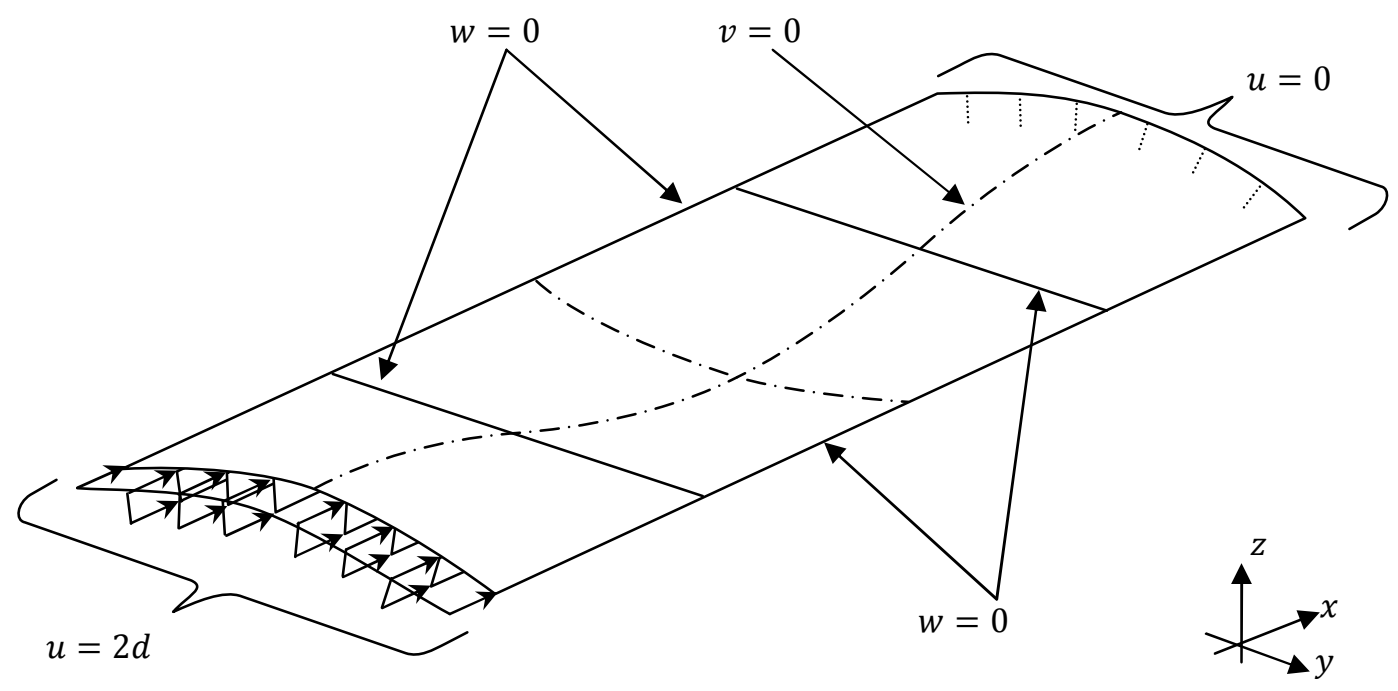

Figure 12: Quad Half-Bay Model and Boundary Conditions

\section{E. Postbuckling Procedure}

Postbuckling calculation in Abaqus is a two stage process because if a constant displacement is applied to a panel with perfect geometry, only axial compression will occur, without any buckling. The first step is to make a buckling calculation of the panel, so the critical buckling mode shape can be applied to the second step model, where a general non-linear solver applies end displacement.

Because the panel is loaded by varying displacement, it is not necessary to use a Riks' solver for the postbuckling analysis. Because the panel does not have a collapse mechanism where the change of displacement is reversed, like happens for the applied force, the general solver is sufficient.

\section{Results}

The double half-bay model has been validated against both previously published results, and the authors' VICONOPT results. As explained in Dawe and Wang's paper, ${ }^{7}$ it has been previously calculated that the effect of including the offsets between the web ends and skin centreline at the junctions has a negligible effect to the buckling characteristics, so for these results, the plates are joined at the centrelines.

A comparison of buckling modes and their buckling loads is presented in Table 1, with values given as multiples of the lowest buckling mode for the panel, the $\lambda_{x}=l / 6$ mode (see Figure 13) that has a critical load of $39.4747 \mathrm{kN}$ at an end displacement of $0.32766 \mathrm{~mm}(420 \mu \varepsilon)$. Values are shown for the lowest eigenvalue of each mode shape, with all but the $m=1$ mode being a local type mode. As the figures show, for buckling, the Abaqus results are very close to the theoretical results from VICONOPT. The greatest difference is for the overall mode, and is the only result where the Abaqus buckling load is lower than the one from VICONOPT is for the overall mode. This discrepancy is explained by the slight difference in mode shape, as seen in Figure 14 below, where the Abaqus mode is not an exact sine wave laterally because of the stiffeners. 


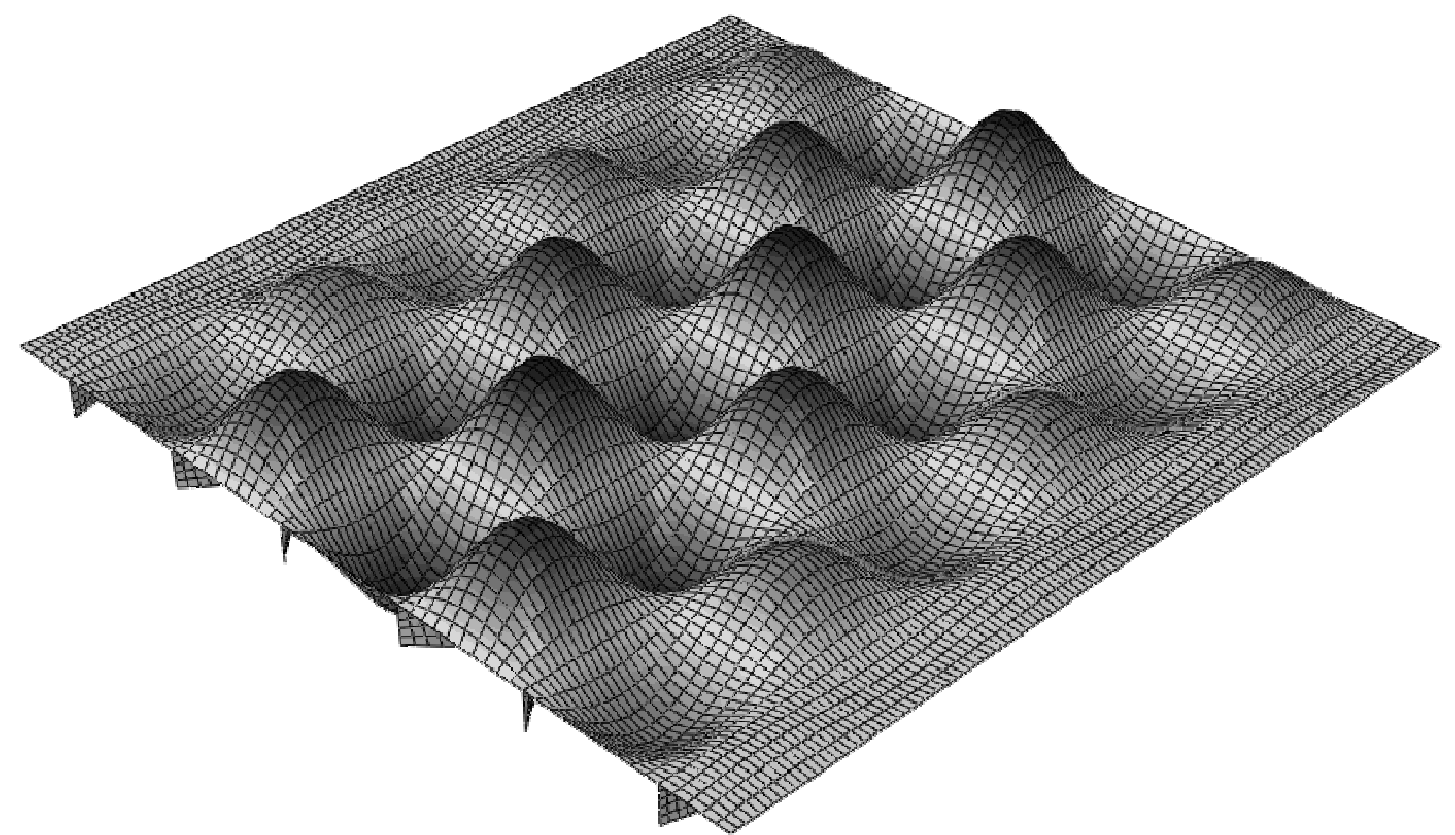

Figure 13: Critical $(m=7)$ Buckling Mode Shape of NASA Example 4 Panel, from Single Bay Model.

Table 1: Comparison of Buckling Loads by Calculation Method.

\begin{tabular}{|c|c|c|c|c|c|}
\hline \multirow{2}{*}{$m$} & \multicolumn{2}{|c|}{ VICONOPT } & \multicolumn{3}{|c|}{ Abaqus, Quad Half-bay Model } \\
\hline & Mode No. & Critical Load Factor & Mode No. & Critical & Load Factor \\
\hline 6 & 1 & 1.0000 & 1 & 1.0002 & $(+0.02 \%)$ \\
\hline 7 & 2 & 1.0112 & 2 & 1.0120 & $(+0.08 \%)$ \\
\hline 5 & 3 & 1.0378 & 5 & 1.0386 & $(+0.08 \%)$ \\
\hline 4 & 9 & 1.1652 & 15 & 1.1683 & $(+0.26 \%)$ \\
\hline 3 & 35 & 1.5001 & 62 & 1.5077 & $(+0.51 \%)$ \\
\hline 2 & 76 & 2.4930 & 138 & 2.5306 & $(+1.51 \%)$ \\
\hline 1 & 85 & 2.8752 & 153 & 2.8079 & $(-2.34 \%)$ \\
\hline
\end{tabular}




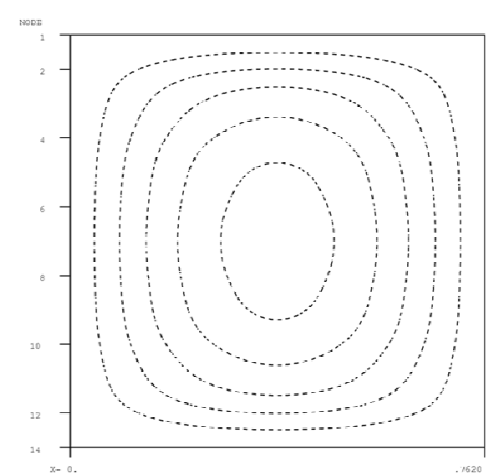

VICONOPT

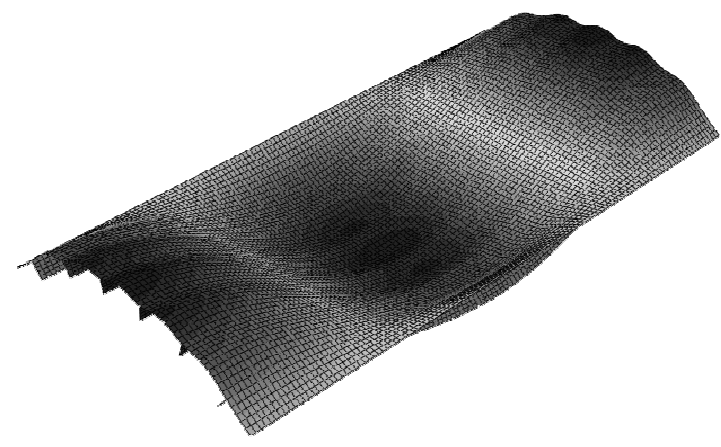

Abaqus, Quad Half-Bay Model

\section{Figure 14: Comparison of $m=1$ Overall Mode Shapes by Calculation Method}

While the modes above show there is a good correlation between modelling methods, when compared by order of criticality, the FEM analysis identifies many more modes than VICONOPT. The $\lambda_{x}=l / 1$ mode is the $85^{\text {th }}$ most critical mode for VICONOPT, and $153^{\text {rd }}$ most critical from FEM. The additional modes shown in the Abaqus results are all possible for an infinite length panel; however, the difference between the result sets can be explained by the assumptions of mode shape in VIPASA. Because the FEM model does not assume or dictate that all modes will be a constant amplitude sine wave, it is not restricted to only identifying these mode types, so it is able to identify additional buckling modes as the one in Figure 15.

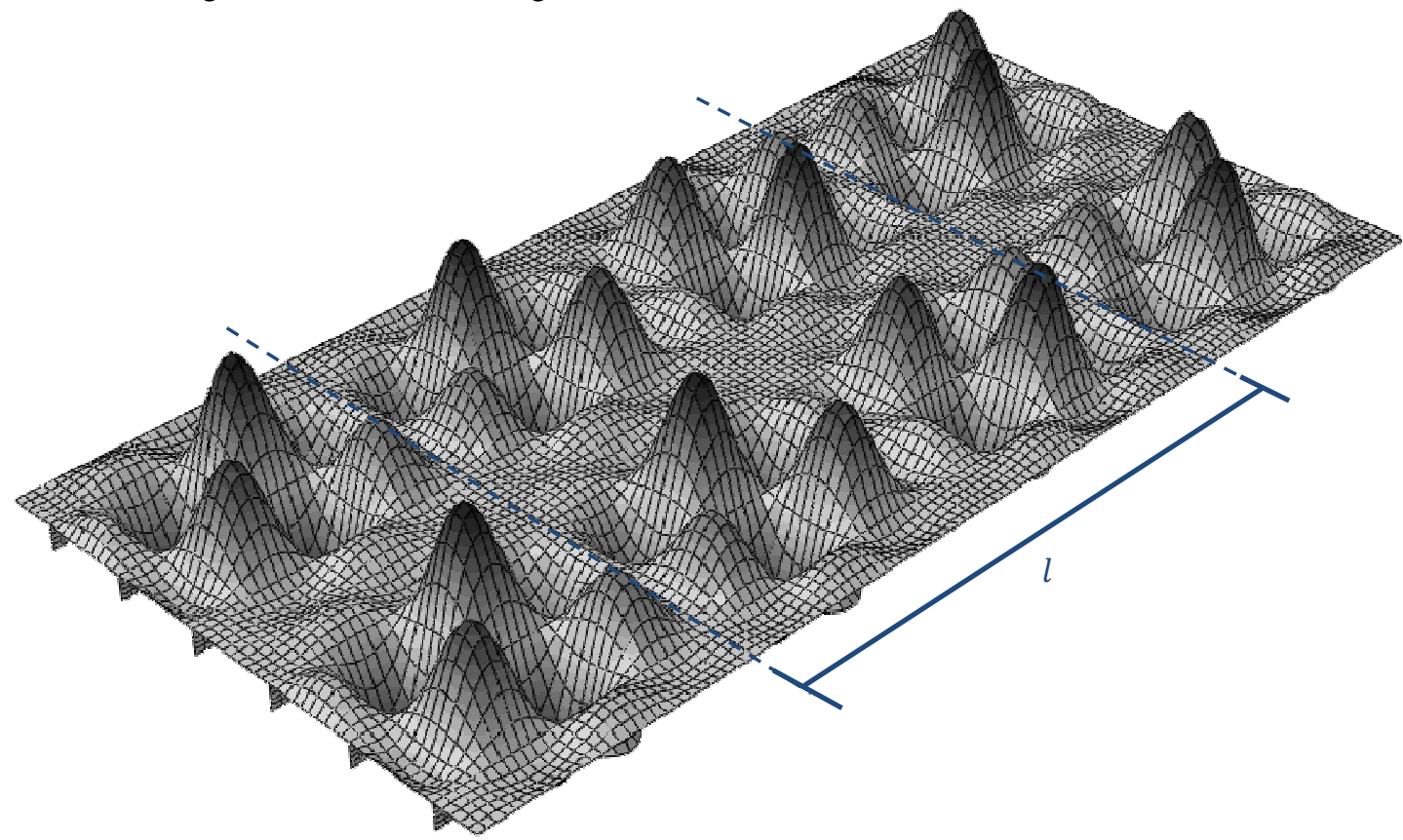

Figure 15: Infinite Plate Buckling Mode without Constant Sine-wave Displacement, from Quad Half-Bay Model (Mode No. 17, Load Factor 1.1850).

When compared to the results from Dawe et al. ${ }^{10}$ the postbuckling behaviour of the FEM model shows very good agreement. The graphs of Figure 16 and Figure 18 are almost identical to those published on page 1022 in Ref.10, including the $l / 7$ having a higher force initially after buckling, as shown in detail in Figure 17. Because Dawe, Lam and Azzizan's results are from an FSM calculation and the postbuckling mode shape is fixed, both the $l / 6$ and $l / 7$ initial mode shapes were tested in Abaqus, however, these results are not forced to remain in the same shape. The $l / 6$ and $l / 7$ modes were seeded onto the panel in separate calculations, and while the results show postbuckling stiffnesses to be the same as the previous results, they also show that the panel does not, as has been previously 
suggested, change into the lowest stiffness mode. For both these results, the panel remained in the seeded mode for initial buckling; only jumping in postbuckling into a collapse mode of $\lambda x=l / 1$ without any intermediate changes. For the $m=6$ seeded results, the panel jumped to the overall mode at 3.05 times critical strain and 2.23 times critical load. The $m=7$ seeded panel was more stable, and entered the overall mode at 4.91 times critical strain and 3.01 times critical load.

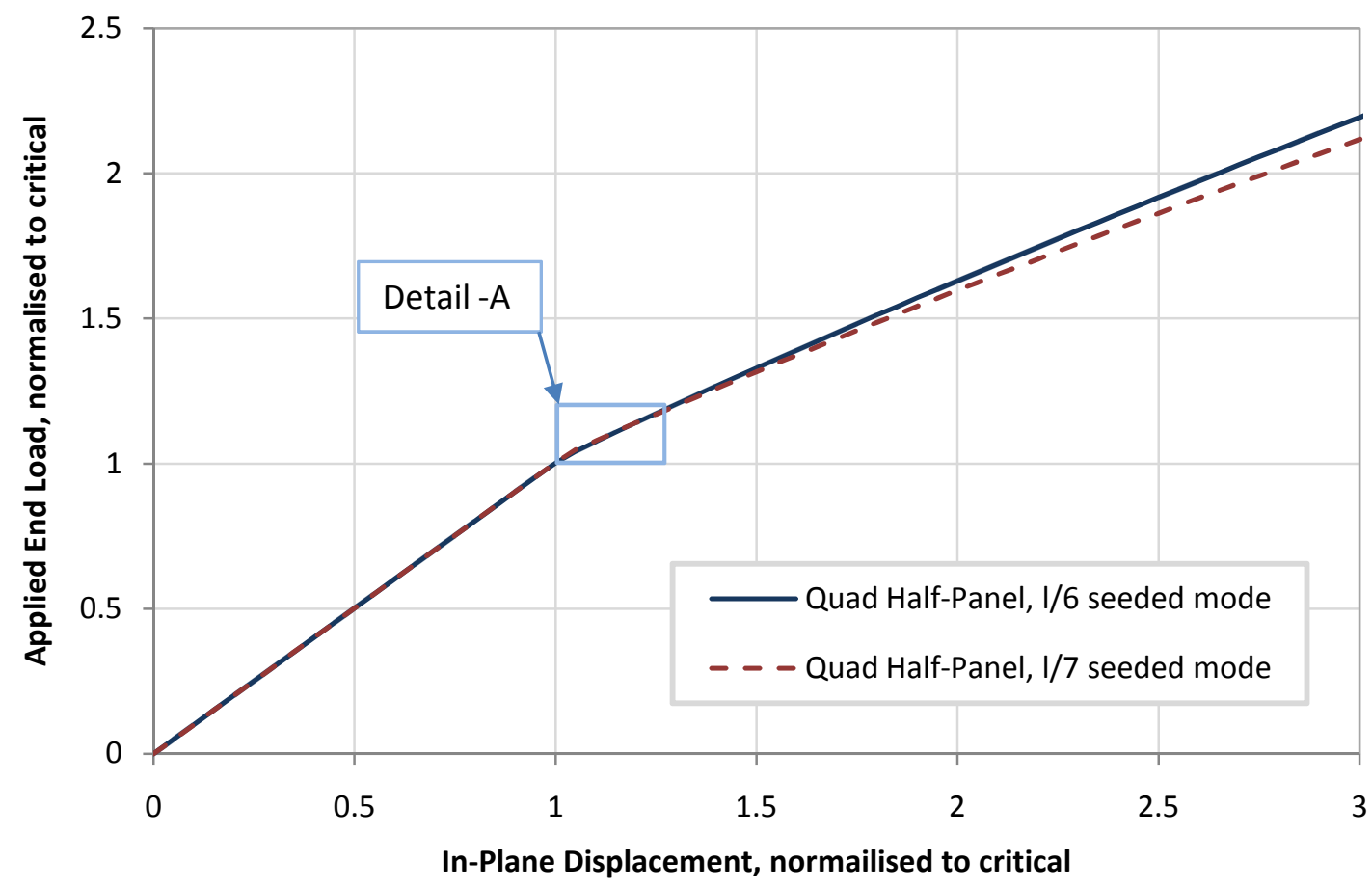

Figure 16: Force vs. In-Plane Displacement from Abaqus Quad Half-Bay Postbuckling.

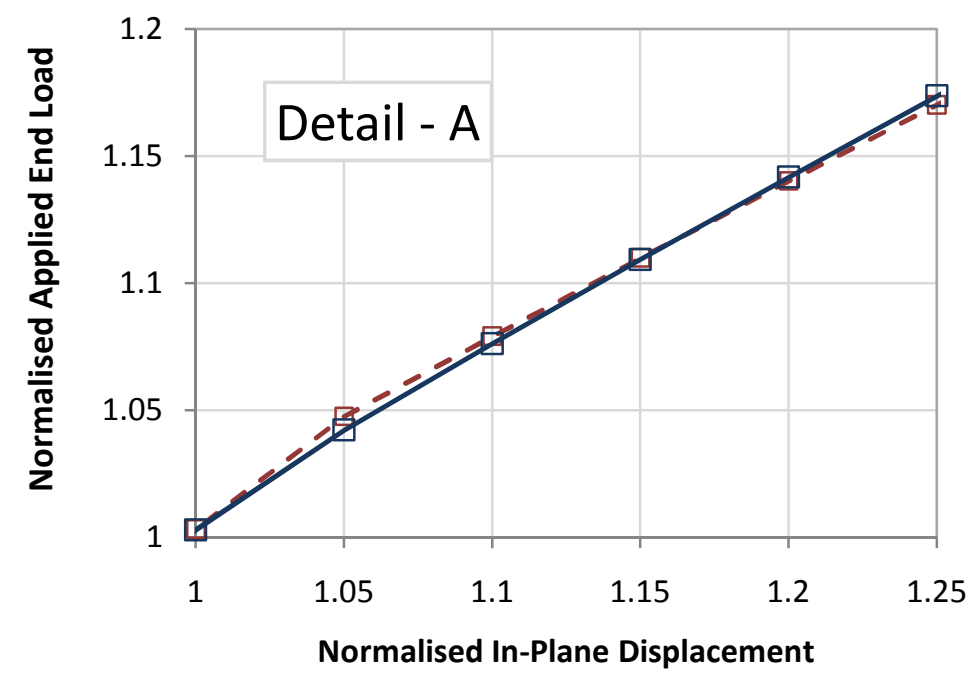

Figure 17: Detail-A from Figure 16.

For the double half-bay model, odd modes will not appear even if mode end-to-end linking is applied; however, they may appear for single bay models and appear, superficially, to be valid modes for the infinite length case. It is not advised however, to apply end-to-end nodal linking to a single bay model even if only local modes are required. For the NASA Example 4 panel, an $l / 7$ mode result was calculated that was not of constant amplitude along the panel length as shown in Figure 19. Because the end-to-end linking forces force the panel bays to be identical, instead of vertically reflected, as for a constant amplitude odd mode, the result is a mode where the boundaries are 
equivalent to fixed ends. When loaded in postbuckling, this mode type will not act like the expected constant amplitude mode as shown in Figure 16. Here the response is of the $l / 7$ single plate mode in area (a) has a different stiffness to the double half-bay model's response, and changes to a continuous $l / 8$ mode soon into the postbuckling region, area (b).

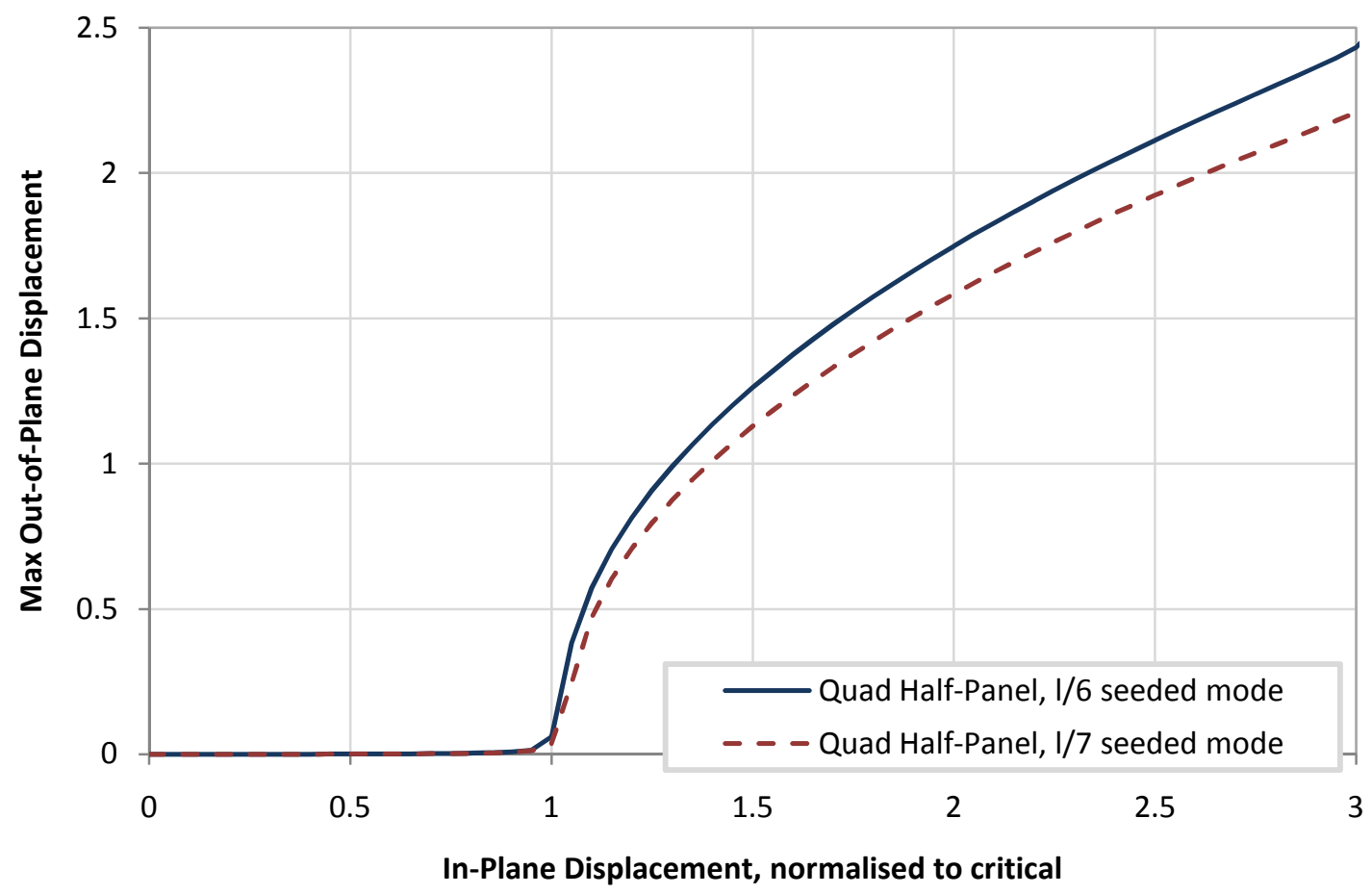

Figure 18: Maximum Skin Out-of-Plane Displacement vs. In-Plane Displacement from Abaqus Quad HalfBay Postbuckling.

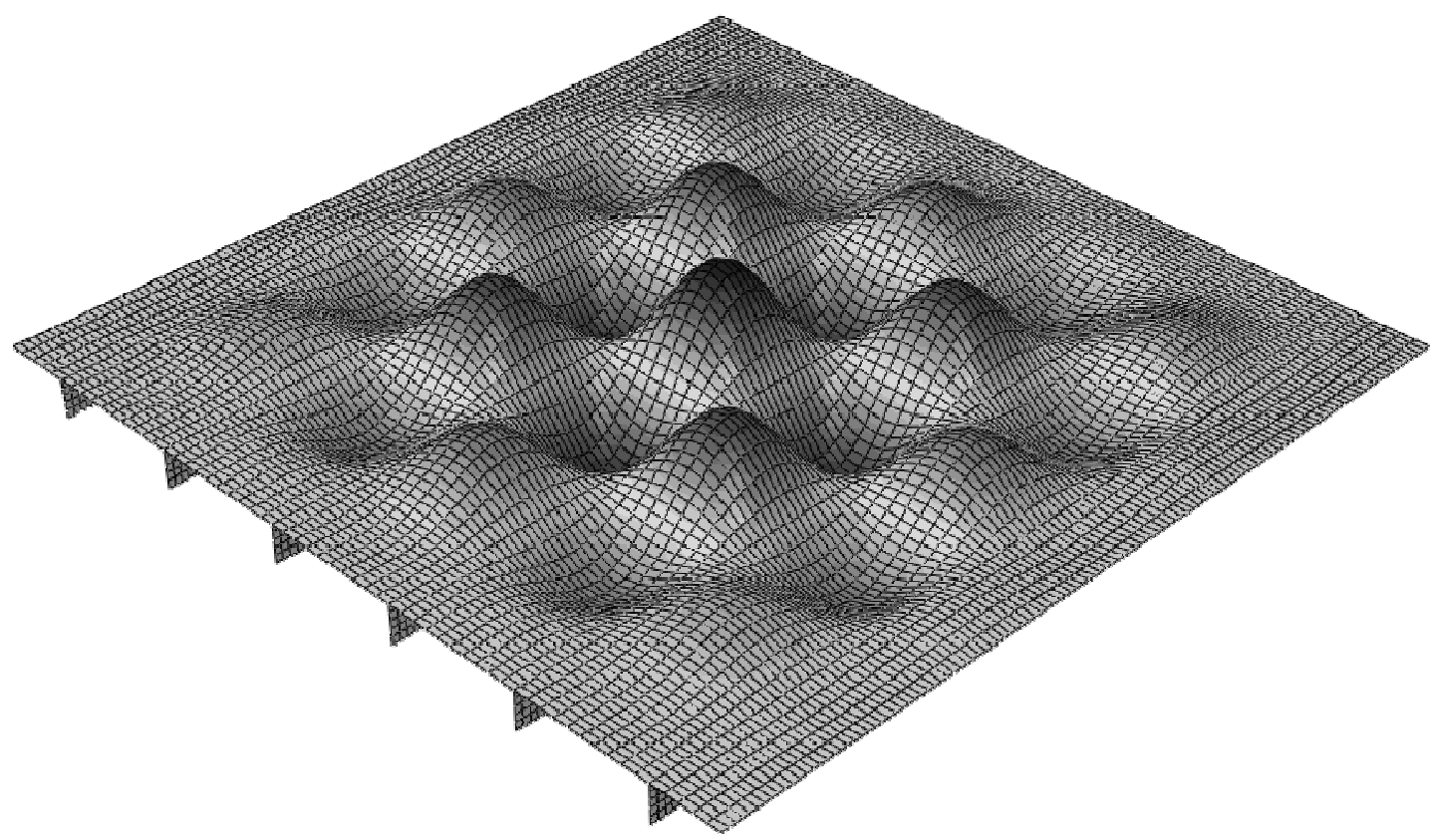

Figure 19: Varying Amplitude $m=7$, Single Bay Mode. 


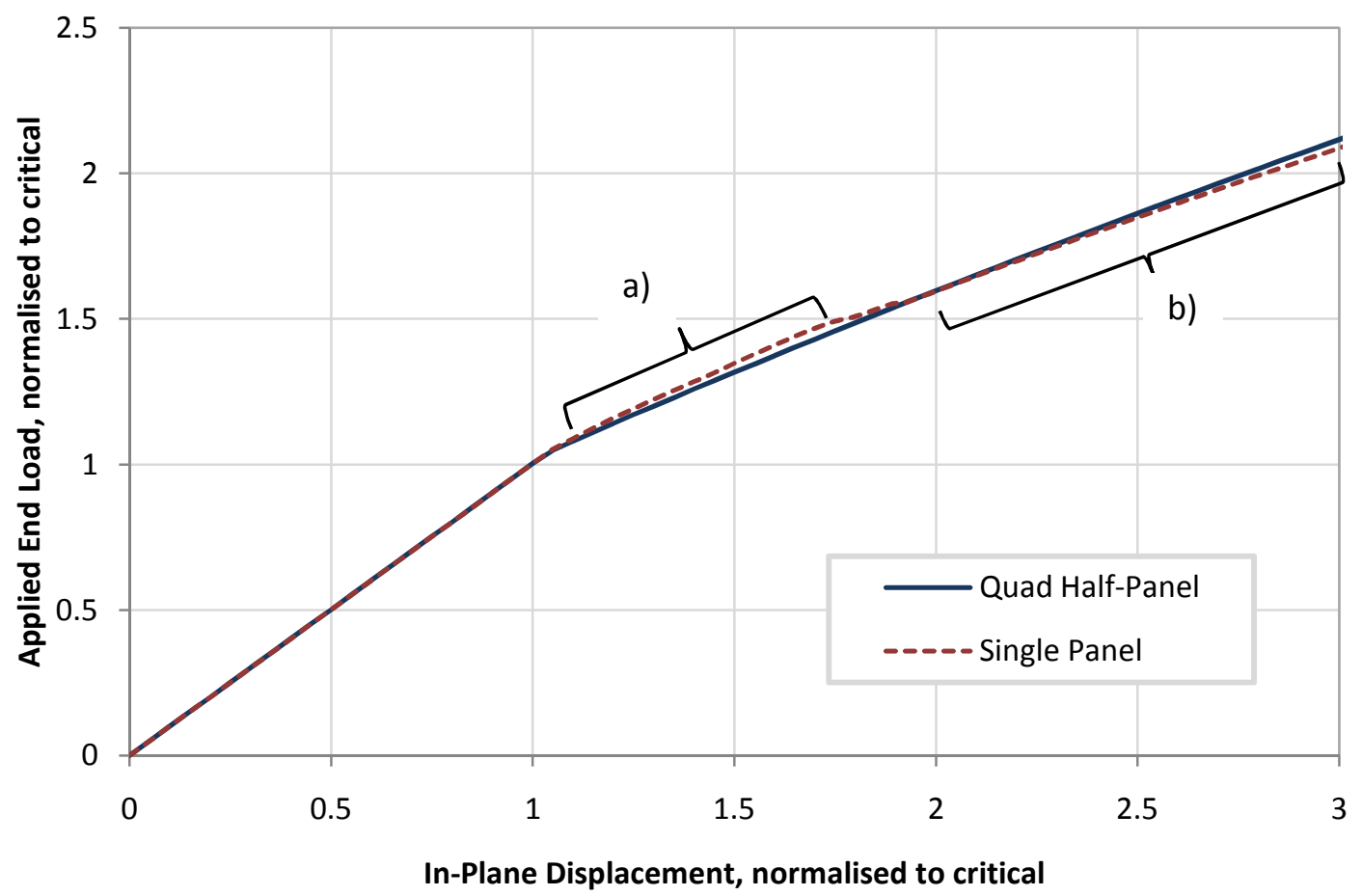

Figure 20: Postbuckling Comparison between Constant and Varying Displacement $\boldsymbol{m}=7$ modes.

\section{Conclusion}

Classical plate theory and its derivatives, like the finite strip method, are built on the assumption that the response of a plate is similar to the response of an infinite length plate, and that the buckling mode will be of a longitudinal and lateral sinusoidal shape. With a finite element model, the buckling mode shape need not be known or prescribed to find the solution.

The finite element modelling process described in this paper has been shown to be accurate compared to FSM results to within a 3\% error when modelling both buckling and postbuckling; and this has been verified against previously published results and methods. The method is shown to be logically valid in its derivation and, while it is only strictly valid for panels where the lowest overall buckling is of a $\lambda x=l / 1$ mode, this is a significant subset of practical panels and could be applied selectively to other conditions.

Being able to model infinite length panels using a method other than FSM or CPT offers a way of verifying the accuracy of results created by these methods, while including the same assumptions of panel behaviour. It is known that calculation of postbuckling in VICONOPT has scope for improvement, and work at Cardiff University continues to this effect, ${ }^{11}$ and this model would be suitable for comparison.

\section{Acknowledgments}

This work is funded with the help of the Engineering and Physical Sciences Research Council and Airbus UK.

\section{References}

${ }^{1}$ Iyengar N.G.R., "Structural Stability of Columns and Plates", Ellis Horwood, 1988.

${ }^{2}$ Watson A., Fenner P., Featherston C., Kennedy D., "Postbuckling Stability of Panels with Torsional Buckling", 50th AIAA/ASME/ASCE/AHS/ASC Structures, Structural Dynamics, and Materials Conference, 2009, AIAA-2009-2510.

${ }^{3}$ Wittrick W.H., "General Sinusoidal Stiffness Matrices for Buckling and Vibration Analysis of Thin Flat-Walled Structures", International Journal of Mechanical Science, Vol. 10, 1968, pp. 949-966.

${ }^{4}$ Henwood D., Bonet J., "Finite Elements: A Gentle Introduction", Macmillan Press, 1996.

${ }^{5}$ Wittrick, W.H., and Williams, F.W., "Buckling and Vibration of Anisotropic or Isotropic Plate Assemblies under Combined Loadings", International Journal of Mechanical Sciences, Vol. 16, No. 4, 1974, pp. 209-239.

${ }^{6}$ Wittrick, W.H., and Williams, F.W., “An Algorithm for Computing Critical Buckling Loads of Elastic Structures", Journal of Structural Mechanics, Vol. 1, No. 4, 1973. pp. 497-518. 
${ }^{7}$ Dawe D.J., Wang S., "Postbuckling Analysis of Composite Laminated Panels", AIAA Journal, Vol. 38, Nov. 2000, No. 11, pp. 2160-2170.

${ }^{8}$ Peskham V., Dawe D.J., "Buckling and Vibration of Finite-Length Composite Plate Structures with Diaphragm Ends, Part II: Computer Programs and Buckling Applications", Computer Methods in Applied Mechanics and Engineering, Vol. 77, 1989, pp. 227-252.

${ }^{9}$ Stroud W.J., Greene W.H., Anderson MS, "Buckling Loads of Stiffened Panels Subjected to Combined Longitudinal Compression and Shear: Results Obtained with PASCO, EAL and STAGS Computer Programs", Computer Methods in Applied Mechanics and Engineering, NASA TP2215, 1984.

${ }^{10}$ Dawe D.J., Lam S.S.E. and Azzizan Z.G., "Finite Strip Post-Local-Buckling Analysis of Composite Prismatic Plate Structures", Computers and Structures, Vol. 48, No. 6, 1993, pp. 1011-1023

${ }^{11}$ Featherston C.A., Kennedy A., Qu S., "Recent Developments in Exact Strip Analysis and Optimum Design of Aerospace Structures", Journal of Mechanical Engineering Science Proceedings Part C, vol. 221(4), 2007, pp. 399-413. 